\title{
Resolvable Spaces and Compactifications
}

\author{
Monerah Al-Hajri, Karim Belaid \\ Department of Mathematics, Faculty of Sciences of Dammam, \\ Girls College, University of Dammam, Dammam, KSA \\ Email: M3sbkh@yahoo.com, belaid412@yahoo.fr, kbelaid@ud.edu.sa
}

Received January 15, 2013; revised February 19, 2013; accepted March 17, 2013

Copyright (C) 2013 Monerah Al-Hajri, Karim Belaid. This is an open access article distributed under the Creative Commons Attribution License, which permits unrestricted use, distribution, and reproduction in any medium, provided the original work is properly cited.

\begin{abstract}
This paper deals with spaces such that their compactification is a resolvable space. A characterization of space such that its one point compactification (resp. Wallman compactification) is a resolvable space is given.
\end{abstract}

Keywords: Resolvable Space; Alexandroff Compactification; Wallman Compactification

\section{Introduction}

In 1943, Hewitt [1] has introduced the notion of resolvable space as follows: A topological space is said to be resolvable if it has two disjoint dense subsets. Hence a topological space $X$ is resolvable if and only if $X$ is written as a union of two disjoint dense subsets. Hewitt in [1] has also called a topological space $X$ maximally irresolvable if each dense subset of $X$ is open. Nowadays, maximally irresolvable spaces are called submaximal spaces.

Recently, Belaid et al. [2], were interested in spaces such that their compactifications are submaximal. They proved that if $X$ is a topological space and $K(X)$ is a compactification of $X$, then the following statements are equivalent:

1) $K(X)$ is submaximal.

2) For each dense subset $D$ of $X$, the following properties hold:

a) $D$ is co-finite in $K(X)$;

b) for each $x \in K(X)-D,\{x\}$ is closed.

It is clear that a compactification of resolvable spaces is resolvable. Hence the following question is natural:

"Characterize spaces $X$ such that a compactification $K(X)$ of $X$ is a resolvable space?"

The first section is devoted to a brief study of spaces $X$ such that their compactification is a resolvable space. The particular case of the one-point compactification is given.

The purpose of the second section is to give an intrinsic topological characterization of spaces $X$ such that the Wallman compactification $w X$ of $X$ is a resolvable space.

\section{Resolvable Space and Compactifications}

First, recall that a compactification of a topological space $X$ is a couple $(K(X), e)$, where $K(X)$ is a compact space and $e: X \rightarrow K(X)$ is a continuous embedding ( $e$ is a continuous one-to-one map and induces a homeomorphism from $X$ onto $e(X))$ such that $e(X)$ is a dense subspace of $K(X)$. When a compactification $(K(X), e)$ of $X$ is given, $X$ will be identified with $e(X)$ and assumed to be dense in $K(X)$.

Let us give some basic facts about space such that its compactification is a resolvable space.

Lemma 2.1 Let $X$ be a topological space, $K(X)$ be a compactification of $X$ and $A$ be a subset of $K(X)$. If $X$ is an open set of $K(X)$, then the following statements are equivalent:

1) $A$ is a dense subset of $K(X)$;

2) $A \cap X$ is a dense subset of $X$.

Proof. 1) $\Rightarrow 2$ ) Let $O$ be an open set of $X$. Since $X$ is an open set of $K(X), O$ is an open set of $K(X)$. Hence $O \cap A \neq \varnothing$. Thus $O \cap(A \cap X) \neq \varnothing$; so that $A \cap X$ is a dense set of $X$.

$2) \Rightarrow 1$ ) Let $U$ be an open set of $K(X)$. Since $U \cap X$ is a non-empty open set of $X$, $(A \cap X) \cap(U \cap X) \neq \varnothing$. Then $U \cap A \neq \varnothing$. Therefore $A$ is a dense set of $K(X)$.

An immediate consequence of Lemma 1.1 is the following.

Proposition 2.2 Let $X$ be a topological space and $K(X)$ be a compactification of $X$. If $X$ is an open set of $K(X)$, then the following statements are equivalent:

1) $X$ is resolvable; 
2) $K(X)$ is resolvable.

Let us recall the construction of the one-point compactification: For any non-compact space $X$ the one-point compactification of $X$ is obtained by adding one extra point $\infty$ (called a point at infinity) and defining the open sets of $\tilde{X}=X \bigcup\{\infty\}$ to be the open sets of $X$ together with the sets of the form $O \bigcup\{\infty\}$, where $O$ is an open set of $X$ such that $X-O$ is a closed compact set of $X$. The one point compactification $\tilde{X}$ of $X$ is also called the Alexandroff compactification of $X$ [3].

The following result characterizes space such that its one point compactification is a resolvable space. Its proof follows immediately from Proposition 2.2; thus it is omitted.

Proposition 2.3 Let $X$ be a non-compact topological space. Then the following statements are equivalent:

1) The one-point compactification $\tilde{X}$ of $X$ is resolvable;

2) $X$ is resolvable.

\section{Resolvable Space and Wallman Compactification}

First, recall that the Wallman compactification of $T_{1}$ space was introduced, in 1938, by Wallman [4] as follows:

Let $\mathcal{P}$ be a class of subsets of a topological space $X$ which is closed under finite intersections and finite unions.

A $\mathcal{P}$-filter on $X$ is a collection $\mathcal{F}$ of nonempty elements of $\mathcal{P}$ with the properties:

1) $\mathcal{F}$ is closed under finite intersections;

2) $P_{1} \in \mathcal{F}, P_{1} \subseteq P_{2}$ implies $P_{2} \in \mathcal{F}$.

A $\mathcal{P}$-ultrafilter is a maximal $\mathcal{P}$-filter. When $\mathcal{P}$ is the class of closed sets of $X$, then the $\mathcal{P}$-filters are called closed filters.

The points of the Wallman compactification $w X$ of a space $X$ are the closed ultrafilters on $X$. For each closed set $D \subseteq X$, define $D^{*}$ to be the set

$D^{*}=\{\mathcal{F} \in w X \mid D \in \mathcal{F}\}$. Thus

$\mathcal{C}=\left\{D^{*} \mid D\right.$ is a closed set of $\left.X\right\}$ is a base for the closed sets of a topology on $w X$.

Let $U$ be an open set of $X$, we define $U^{*}=\{\mathcal{F} \in w X \mid F \subseteq U$ for some $F$ in $\mathcal{F}\}$, it is easily seen that the class $\left\{U^{*} U\right.$ is an open set of $\left.X\right\}$ is a base for open sets of the topology of $w X$. The following properties of $w X$ are frequently useful:

Proposition 3.1 Let $X$ be a $T_{1}$-space and $w X$ the Wallman compactification of $X$. Then the following statements hold:

1) $w X$ is a $T_{1}$-space;

2) For $x \in X$ and

$\Phi(x)=\{F \mid F$ is a closed set of $X$ and $x \in F\}$. Then $\Phi$ is an embedding of $X$ into $w X \quad(\Phi(x)$ will be identified to $x$ ).

3) If $U$ is an open set of $X$, then $w X-U^{*}=(X-U)^{*}$.

4) If $U_{1}$ and $U_{2}$ are two open sets of $X$, then $\left(U_{1} \cap U_{2}\right)^{*}=U_{1}^{*} \cap U_{2}^{*}$ and $\left(U_{1} \cup U_{2}\right)^{*}=U_{1}^{*} \cup U_{2}^{*}$.

Recall that Kovar in [5] has characterized space with finite Wallman compactification remainder as following:

Proposition 3.2 Let $X$ be a $T_{1}$-space. Then the following statements are equivalent:

1) $\operatorname{Card}(w X-X)=n$;

2) There exists a collection of $n$ pairwise disjoint non-compact closed sets of $X$ and every family of noncompact pairwise disjoint closed sets of $X$ contain at most $n$ elements.

The following proposition follows immediately from Proposition 3.2 and Proposition 3.1-1).

Proposition 3.3 Let $X$ be a $T_{1}$-space and $n \in \mathbb{N}$ such that every family of non-compact pairwise disjoint closed sets of $X$ contains at most $n$ elements. Then $X$ is resolvable if and only if $w X$ is resolvable.

The following lemma has been given in [2] as Remark 4.5 and Remark 4.9.

Lemma 3.4 Let $X$ be a $T_{1}$-space. Then the following properties hold:

1) If $F$ is a closed non-compact subset of $X$, then there exists $\mathcal{F} \in w X-X$ such that $F \in \mathcal{F}$.

2) $\mathcal{F} \in w X-X$. Then for each $F \in \mathcal{F}, F$ is a non-compact closed set of $X$.

The following result is an immediate consequence of Lemma 3.4.

Corollary 3.5 Let $X$ be a $T_{1}$-space, $w X$ be the Wallman compactification of $X$ and $U$ be an open set of $X$. Then the following statements are equivalent:

1) $U \subsetneq U^{*}$;

2) There exists a non compact closed set $F$ of $X$ such that $F \subseteq U$.

Now, we are in a position to give a characterization of spaces such that their Wallman compactification is resolvable.

Theorem 3.6 Let $X$ be a $T_{1}$-space. Then the following statements are equivalent:

1) The Wallman compactification $w X$ of $X$ is resolvable;

2) There exist two disjoint subsets $D_{1}$ and $D_{2}$ of $X$ such that:

a) $X=D_{1} \cup D_{2}$.

b) For $i \in\{1,2\}$ and for each non empty open set $O \subseteq D_{i}$, there exists a non compact closed set $F$ of $X$ such that $F \subseteq O$.

Proof. 1) $\Rightarrow 2$ ) Since $w X$ is a resolvable space, there exist two disjoint dense sets $A_{1}$ and $A_{2}$ of $w X$ such that $w X$ is the union of $A_{1}$ and $A_{2}$. Set $D_{1}=X \cap A_{1}$ and $D_{2}=X \cap A_{2}$. 
Let $i \in\{1,2\}$ and $O$ be a non empty open set of $X$ such that $O \subseteq D_{i}$. Set $j \in\{1,2\}$ such that $j \neq i$. Since $A_{j}$ is a dense subset of $w X, O^{*} \cap A_{j} \neq \varnothing$. Now, $O^{*} \cap\left(A_{j} \cap X\right)=O \cap D_{j}=\varnothing$ implies that $O^{*} \cap\left(A_{j} \cap w X-X\right) \neq \varnothing$. It follows that there exists $\mathcal{F} \in O^{*} \cap\left(A_{j} \cap w X-X\right)$, and thus $O \subsetneq O^{*}$. According to Corollary C4 there exists a non compact closed set $F$ of $X$ such that $F \in \mathcal{F}$ and $F \subseteq O$.

2) $\Rightarrow 1$ ) Let $D_{1}, D_{2}$ be two disjoint subsets of $X$ satisfying the condition b) and such that $X=D_{1} \cup D_{2}$. Let $i \neq j$ in $\{1,2\}$ and we define

$$
\begin{aligned}
& A_{i}=D_{i} \cup\{\mathcal{F} \in w X-X \mid \text { there exists } F \in \mathcal{F} \\
& \text { and an open set } \left.O \text { of } X \text { such that } F \subseteq O \subseteq D_{j}\right\} .
\end{aligned}
$$

It is immediate that $A_{i} \cap A_{j}=\varnothing$.

Now, let $U$ be a open set of $X$. We consider two cases:

Case 1: $U \cap D_{i} \neq \varnothing$. Then $U \cap A_{i} \neq \varnothing$. So $U^{*} \cap A_{i} \neq \varnothing$.

Case 2: $U \cap D_{i}=\varnothing$. Then $U \subseteq D_{j}$. By condition b), there exists a non compact closed $F$ of $X$ such that $F \subseteq U$. Let $\mathcal{F} \in w X-X$ such that $F \in \mathcal{F}$. Hence $\mathcal{F} \in U^{*} \cap A_{i}$. Thus $U^{*} \cap A_{i} \neq \varnothing$.

Therefore $A_{i}$ is a dense set of $w X$; so that $w X$ is a resolvable space.

Example 3.7 Let $\mathbb{Q}$ be the set of all rational numbers equipped with the natural topology $T_{\mathbb{Q}}$. Let $X=\mathbb{Q} \times\{0\} \cup \mathbb{Q} \times\{1\}$ equipped with the topology $T=\left\{U \times\{0\} \mid U \in T_{\mathbb{Q}}\right\} \cup\left\{\mathbb{V} \times\{1\} \mid V \in T_{\mathbb{Q}}\right\}$. It is immediate that the topological space $X$ satisfies the condition 2) of the Theorem 2.6. Then $w X$ is a resolvable space. The previous result incites us to ask the following question.

Question 3.8 Let $X$ be a space. We denote by $\beta_{\omega} X$ (resp. $\beta X$ ) the $T_{0}$-compactifcation of $X$ introduced by Herrlich in [6] (resp. the Stone Cech compactification). When is $\beta_{\omega} X$ (resp. $\left.\beta X\right)$ a resolvable space?

\section{Acknowledgements}

This paper has been supported by deanship of scientific research of University of Dammam under the reference 2011085.

\section{REFERENCES}

[1] E. Hewitt, “A Problem of Set Theoretic Topology,” Duke Mathematical Journal, Vol. 10, No. 2, 1943, pp. 309-333. doi:10.1215/S0012-7094-43-01029-4

[2] K. Belaid, L. Dridi and O. Echi, "Submaximal and Door Compactifications," Topology and Its Applications, Vol. 158, No. 15, 2011, pp. 1969-1975. doi:10.1016/j.topol.2011.06.039

[3] J. L. Kelly, “General Topology,” D. Van. Nostrand Company, Inc., Princeton, 1955.

[4] H. Wallman, "Lattices and Topological Spaces," Annals of Mathematics, Vol. 39, No. 1, 1938, pp. 112-126. doi:10.2307/1968717

[5] M. M. Kovar, "Which Topological Spaces Have a Weak Reflection in Compact Spaces?” Commentationes Mathematicae Universitatis Carolinae, Vol. 36, No. 3, 1995, pp. 529-536.

[6] H. Herrlich, "Compact $T_{0}$-Spaces and $T_{0}$-Compactifications,” Applied Categorical Structures, Vol. 1, No. 1, 1993, pp. 111-132. doi:10.1007/BF00872990 